\title{
Cloud Agile Manufacturing
}

\author{
Francisco Macia-Perez ${ }^{1}$, Jose Vicente Berna-Martinez ${ }^{1}$, Diego Marcos-Jorquera ${ }^{1}$, \\ Iren Lorenzo-Fonseca ${ }^{1}$, Antonio Ferrandiz-Colmeiro ${ }^{1}$ \\ ${ }^{I}$ (Department of Computer Science, University of Alicante)
}

\begin{abstract}
This paper proposes a new manufacturing paradigm, we call Cloud Agile Manufacturing, and whose principal objective is to offer industrial production systems as a service. Thus users can access any functionality available in the cloud of manufacturing (process design, production, management, business integration, factories virtualization, etc.) without knowledge - or at least without having to be experts — in managing the required resources. The proposal takes advantage of many of the benefits that can offer technologies and models like: Business Process Management (BPM), Cloud Computing, Service Oriented Architectures (SOA) and Ontologies. To develop the proposal has been taken as a starting point the Semantic Industrial Machinery as a Service (SIMaaS) proposed in previous work. This proposal facilitates the effective integration of industrial machinery in a computing environment, offering it as a network service. The work also includes an analysis of the benefits and disadvantages of the proposal.
\end{abstract}

Keywords - Agile Manufacturing, Cloud Computing, Business Process Management, Semantic Industrial Machinery

\section{INTRODUCTION}

Internet has given customers the ability to select the consumer articles that suit them best at all times and prices that best fit their capabilities. This is driving manufacturing organizations to evolve from traditional models oriented to mass production towards models that facilitate mass customization [1] but without losing the advantages of economy of scale provided the classical models. This new scenario requires new patterns organizations of production, more dynamic and flexible, which can adapt flexibly to the changes occurring in the environment due to market demand [2] [3] without causing a major impact on the final costs of the products.

To meet the demands of the new models, the paradigm Business Process Management (BPM) has become the focus of business process management best suited [1] [3], because BPM provides continuous change as one of its main characteristics and facilitates the adaptation of agile business processes and IT infrastructures that support them.

The implementation of a comprehensive BPM paradigm is very complicated in the environment of manufacturing organizations because there is a conceptual and technological gap between the levels of business management and production. This gap is caused mainly by the high stiffness of manufacturing elements located in the lower levels of production [4]. In previous work we have proposed a vision of Industrial Machinery and Service (se call IMaaS - Industrial Machinery as a Service) [5] [6], integrated under a paradigm of Service Oriented Architecture (SOA). This proposal has been successful to solve the problem of gap mentioned, enabling you to model the manufacturing processes of industrial machinery as a part of the System of Business Process Management (BPMS) [3].

By eliminating this gap opens up a whole range of possibilities in the management of processes in manufacturing organizations. However, this range of possibilities now moves the traditional bottleneck between the levels of enterprise and plant to the domain of modeling and process management [7].

To alleviate this burden, in another later work [7] we proposed a system that automates the modeling process and composition according to the organization's strategic objectives, delegating to system the subsequent deployment and execution in different manufacturing organizations. In this way, processes can be defined independently of the physical structure of the factory and can be adapted flexibly to existing resources at all times. To do this, the proposal is based on the synergy between IMaaS model [6] together with the incorporation of knowledge in the definition of processes and services involved, using ontologies described in [7] [8]. This proposal is called Semantic Industrial Machinery and Service (SIMaaS - Semantic IMaaS).

From SIMaaS concept, we can go one step further in the concept of Agile Manufacturing for solving the problem of outsourcing the manufacturing processes through an integrated supply chain [9].

From the viewpoint of process management, the concepts defined in the Cloud Computing paradigm [10] provide the tools to develop the techniques of Integral Management of Supply Chain [9]. However, this paradigm has been defined to provide support for computational elements, leaving outside its management the elements of production, and this problem is solved with SIMaaS.

All in all, based on the concept of SIMaaS and Cloud Computing, this paper proposes the creation of a new manufacturing paradigm, we call Cloud Agile Manufacturing. The main objective of this proposal is that industrial production systems can be offered as a service, so that users can access to any functionality available in the cloud of manufacturing (process design, production, management, business integration, factories virtualization, etc.) without knowledge - or at least without having to be experts - in managing the required resources.

The proposed paradigm draws on the model of layers of cloud computing to which, in a synthesized form, incorporates a layer above responsible for the management 
of production processes as a service and another layer, this time below, responsible for industrial machinery. The distinguishing feature of our approach over other approaches [11] is that it is built on the validated concept SIMaaS. In fact, the working method is to break this concept to be placing the resulting components in the different layers of the new model of Cloud Agile Manufacturing proposed.

\section{CLOUD AGILE MANUFACTURING}

Our proposal of Cloud Agile Manufacturing is a manufacturing paradigm in which: all elements offered by an industrial system are in the form of service, Thus, users can access all services available in the cloud of manufacture, without knowledge (or at least not experts) in the management of resources used.

The concept is built on Internet technologies and is largely based on the paradigm of Cloud Computing. Following its principles, technological infrastructure incorporates the Software as a Service (SaaS) and related concepts, combining them with our proposed Semantic Industrial Machinery and Service (SIMaaS) [7] [8], Thus, the system can deliver capabilities of Agile Manufacturing and Supply Chain Management (SCM), relying on the resources of the Internet for this.

Thus, one can also define Cloud Agile Manufacturing from this point of view: as a new model of manufacturing services, infrastructure and technology that allows users access to a catalog of standardized services and meet the needs of your business, in a flexible and adaptive form, in case of unforeseen demand or peak workloads, paying only for consumption made.

The paradigm shift that provides agile manufacturing in the cloud is the basis of the potential increase in the number of services based on industrial production on Internet. This creates benefits for both manufacturers (constituted in manufacturing services providers), who can provide more rapidly and efficiently a large number of services, and users (service consumers) who are able to access them enjoying the transparency and immediacy of the system, a comprehensive and unified management of their supply chain and a pay per use.

Cloud Agile Manufacturing gets to bring these advantages based on a dynamic IT infrastructure that is characterized, among other factor: by a high degree of automation, rapid mobilization and high resource utilization, high adaptability to meet variable demand, and advanced virtualization industrial machinery, factories, production systems and a flexible cost based in consumption.

\section{LAYERS AND COMPONENTS}

The proposed paradigm can be easily structured in layers following the principles set by Cloud Computing, but with particular emphasis on the concept of Manufacture or Process as a Service and incorporating the concept of Machine as a Service.

The solution proposed in this study (summarized in Figure 1) is developed using a methodology that consists of five major stages: (1) define the two new layers to manage the new concepts that are incorporated and that will characterize the new paradigm Process and Machine (layer
PraaS and layer ImaaS, fig. 1) and incorporate to the traditional model Cloud Computing; (2) study in depth the concept of Semantic Industrial Machinery as a Service (SIMaaS); (3) break it down into layers according to the levels at which it was conceived: from mechanical and hardware levels to the services and processes; (4) redistributing these levels between the set of layers of Cloud Agile Manufacturing by their nature and functionality; and (5) redefine existing layers in the traditional model (SaaS, PaaS, IaaS) so as to accommodate new services and actors involved in each of them.

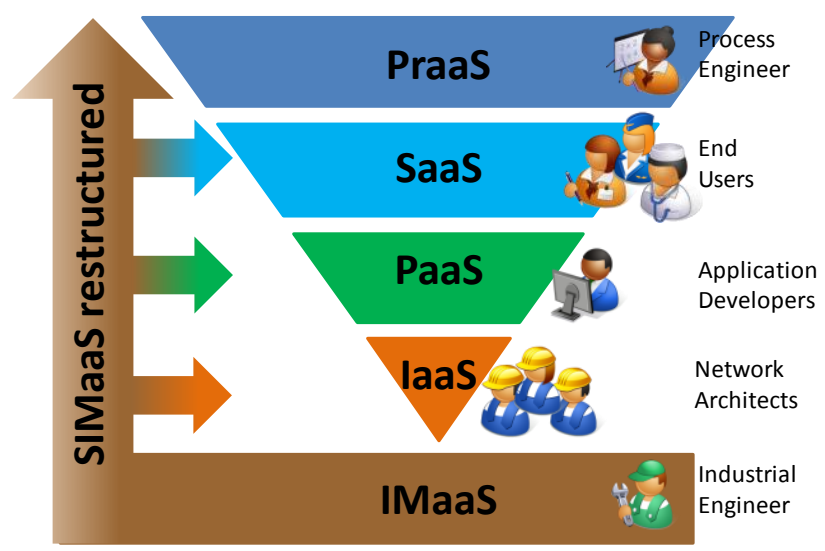

Fig.1. Layers of Cloud Agile Manufacturing paradigm.

As can be seen in Fig. 1, the greater part of the intermediate layers correspond to the paradigm Cloud Computing. These layers have to be redefined to conform to the characteristics of new paradigm, as reflected in stage 5 of the methodology described. But in essence, have very similar functionality to the original, so that (mostly for reasons of space) we will not re-define them in this document.

Accordingly, newer layers are precisely the first PraaS (Production as a Service), and last IMaaS (Industrial Machinery as a Service). The following sections describes both layers.

\section{Praas - Production as a Service}

The concept PraaS already defined in some specific contexts of Cloud Computing, and from the functional point of view, is very similar to the existing proposals. However, from the practical point of view, the philosophy behind our work goes far beyond the current definitions. The fundamental difference is that today, the objective of this level is focused on management and organization processes. Thus, after defining the processes, the system cannot guarantee to be executed on a specific industrial machinery and will not have access to the result of the execution (because the cloud really does not provide the physical, mechanical and electrical industrial machinery). Using our approach, is also focused on its deployment, execution and control. In our proposal, once selected industrial machinery and processes defined, can be deployed and run on the cloud, transparently (because our proposal provides industrial machinery in all its dimensions. This means that the system will have to 
orchestrate all manufacturing resources required to achieve the deployment of production process.

\section{IMAAS - INDUSTRIAL MACHINERY AS A SERVICE:}

We define this layer based on the concept of SIMaaS [7] [8]. Thus the Industrial Machinery is incorporated into the cloud as infrastructure and manufacturing resources, similar to how they do IT infrastructures through IaaS layer. The main technology applied to design of this layer is virtualization. In this case, simple machine virtualization , complex machinery virtualization, virtualization of sections of factories, virtualization of factory and virtualization of factory sets. Of course, each of which can be defined separately for subsequently be combined in the definition of a virtual set larger.

In addition to the virtualization of factories, this layer should handle the use of production resources, This layer should provide features as dynamic adaptation to changes (such as peak demand), optimizing the use of resources by balancing the workload between them and access to quality metrics, process control and status of resources, raw materials and processed products..

\section{Models of Cloud Manufacturing}

Following the paradigm of cloud computing, we can distinguish three types of clouds agile manufacturing: public, private and hybrid.

Public clouds are handled by third parties, and the work of many different clients may be mixed in the factories (virtual), servers, storage systems and other infrastructure in the cloud. End users do not know what other clients works may be carried out in the same factories, even on the same machines. In this type of cloud, the end user basically deals only edit their processes and track them.

Private clouds are a good choice for companies that need high data protection and editing at lower levels (the process) of the services offered by cloud: definition of its own services, computational resources and production, machine or, even, factories. Private clouds are in demand infrastructure managed by a single customer who controls what and where you run a process. They are owners of the factory, of IT and can decide which users are allowed to use the infrastructure.

Hybrid clouds combine the models of public and private clouds. Every customer is a part owner and shares another, albeit in a controlled manner. Hybrid clouds may be the key to achieving an external supply in scale form and under demand, but these clouds add the complexity of determining how to allocate tasks and processes across these different environments. Companies may feel some attraction to the promise of a hybrid cloud, but this option, at least initially, will probably be reserved for simple applications without conditions, which do not require any synchronization or not require highly specialized or expensive equipment.

\section{BENEFITS AND DISAdVANTAgeS OF THE PROPOSAL}

This section critically analyzes the benefits and disadvantages that can present this proposal and collected some of the most widespread controversies.

Some of the main benefits are an extrapolation of those achieved by the Cloud Computing paradigm (see fig. 2) and can be summarized: scalability and economies of scale, the range of choices and agility to manage change, encapsulating change management or that this proposal is being built on the new generation of architectures. But more concrete benefits for the case of agile manufacturing, we find that the new paradigm has great potential and expectations. Among the most important, both from a technical standpoint and from a technological and organizational perspective and conceptual, we highlight the following:

An approach based entirely on Cloud Agile Manufacturing can take advantage of the manufacturing resources of the organization. Today, organizations stand at $15 \%$ or $20 \%$ utilization of IT resources, using cloud can reach up to $40 \%$. Furthermore, we do not need to invest in industrial machinery and, therefore, reduces investment, risk and time for starting the business.

\section{Pros and Cons}

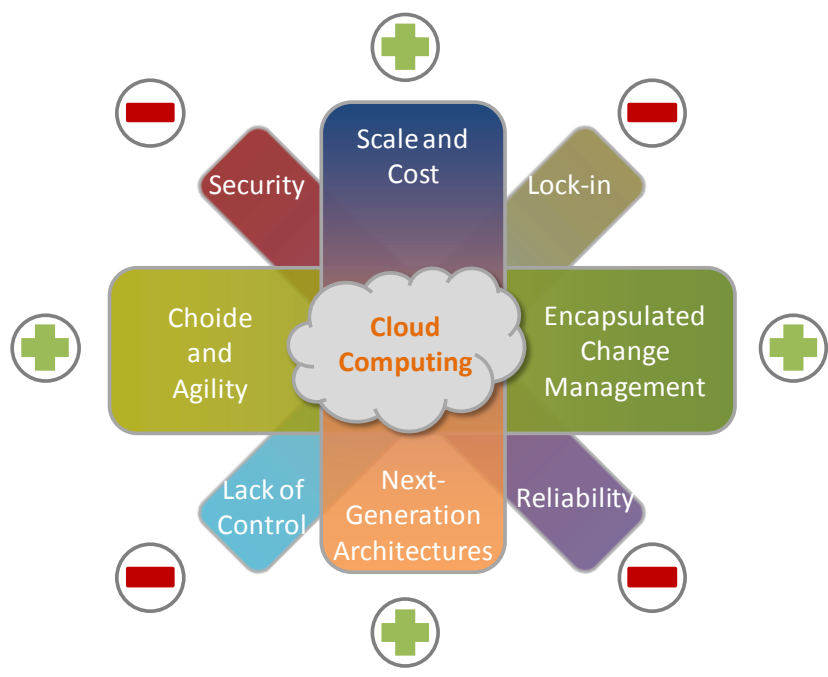

Fig. 2. Advantages and disadvantages of cloud computing paradigm in which the proposal is based.

- The use of resources along with the service delivery models will immediately translate in lower costs for all actors involved: a manufacturer, that provides manufacturing services, obtains revenue by using machines that have vacant, and a manufacturer that access to these services will save the cost of purchasing and maintaining expensive machinery, specialized or required only in a score.

- Also, and laterally, the model facilitates efficient use of energy because it has a more comprehensive control over the operation of machinery.

- The systems developed under the paradigm of Cloud Agile Manufacturing can adapt more easily, speed and lower costs to market needs. Even these systems may face drastic changes in the forms of manufacturing or 
manufactured products and, especially, can address novel business models for exploitation both new services and new products.

- From the point of view of the organization, the paradigm provides outsourcing and integrated supply chain. Thus, each organization involved in product development can focus on those aspects in which specializes.

- It also facilitates the specialization of the manufacturers who may have complex expensive machinery or factories to develop certain products or subproducts for other manufacturers.

- Finally, Cloud Agile Manufacturing allows easy integration, if not almost immediately, of applications and processes both within an organization and between different organizations that wish to collaborate.

However, we also extrapolated the disadvantages of cloud computing. Some of the greatest concern to the community (fig. 2) are: security problems, loss of control (infrastructure, services, management), dependence on a manufacturer, technology, difficulty migrating to other platforms or clouds, and loss of reliability.

In addition to the possible disadvantages and possibly by the novelty of the proposal, there is now an open controversy with this model and similar strategies in which IT has a starring role and take on an importance that was relegated to other sectors plane near the electrical, mechanical or even electronic. However, such proposals, despite the obvious and important gaps that still presented, precisely result of their novelty, are unstoppable, just as in other areas both economic and social have been, because the benefits of new technologies are unquestionable today, not to say indispensable in certain sectors, such as the present case of agile manufacturing.

\section{CONCLUSIONES}

In this paper we have presented a new manufacturing paradigm called Cloud Agile Manufacturing. This paradigm is based on the Internet and especially the concept of Cloud Computing. To ensure the practical feasibility of the proposal, the work has been developed using the concept of Semantic Industrial Machinery as a Service (SIMaaS) developed in previous work. SIMaaS allows us to offer resources of element of production and manufacturing processes through semantic and agile manufacturing using Industrial Machinery as a Service (IMaaS). IMaaS enables us to overcome the technological and conceptual gap between the plant level and management level through service oriented architectures.

The systems developed under this paradigm provide significant benefits for manufacturers who need to deploy agile manufacturing model.

This is an ambitious work that is at an early stage, so many questions both conceptual and applied must be solved. We are currently developing the layers described. We use a complete use case and close to the real problems of the industry so that it can serve as validation and, above all, to provide the necessary feedback to refine and adjust the concepts that are being defined.

\section{REFERENCES}

[1] J. Jeston and J. Neils. Business Process Management. Practical guide to successful implementations. Elsevier, 2006.

[2] H. Smith and P. Fingar. Business Process Management. The Third Wave. Meghan-Kiffer, 2002.

[3] J. F. Chang. Business Process Management Systems. Strategy and Implementation. Auerbach Publications, 2005.

[4] S. L. S. Worthington, W. Boyes. E-Business in Manufacturing: Putting the Internet to work in the industrial enterprise. ISA Press, 2002.

[5] V. Gilart-Iglesias, F. Maciá-Pérez, D. MarcosJorquera and F. J.Mora-Gimeno. Industrial Machine as a Service: Modelling industrial machinery processes. Proc. of 5th International IEEE Conference on Industrial Informatics (INDIN'07). Vienna, 2007.

[6] V. Gilart-Iglesias, F. Maciá-Pérez, J.A. GilMartínez-Abarca and A. Capella-D'alton. Industrial Machines as a Service: A model based on embedded devices and Web Services. Proc. of 4th International IEEE Conference on Industrial Informatics (INDIN'06). Singapore, 2006.

[7] A. Ferrándiz-Colmeiro; V. Gilart-Iglesias; F. MaciáPérez. Semantic Processes Modeling Independent of Manufacturing Infrastructures. IEEE International Conference on Emerging Technologies and Factory Automation (ETFA), 2010 Conference on, pp. 1-8, Bilbao, Spain, Sept. 2010.

[8] F. Macia-Perez; V. Gilart-Iglesias; A. FerrándizColmeiro; J.V Berna-Martinez; J. Gea-Martinez. New Models of Agile Manufacturing Assisted by Semantic. Proceedings of the IEEE EDOC 2009 workshops and short papers, pp. 336-343, Auckland 2009.

[9] M. Christopher, The agile supply chain. Industrial Marketing Management, Industrial Marketing Management. Vol. 29, Issue 1, pp. 37-44, January 2000

[10] A. Weiss, Computing in the Clouds. netWorker, Vol. 11 , no. 4, pp. 16-25, 2007

[11] F, Tao; L Zhang, VC Venkatesh, YL Luo, Y Cheng. Cloud manufacturing: a computing and serviceoriented manufacturing model. Proceedings of the Institution of Mechanical Engineers, Part B, Journal of Engineering Manufacture. 2011.

[12] A. Gunasakaran, Agile manufacturing: A framework for research and development. International Journal of Producting Economics, Vol. 62, Issues 1-2, pp. 87-105, May 1999.

[13] G. Gilder, The information factories, Wired, Vol. 14, no. 10, October 2006. 